\title{
Fully Discrete Energy Stable High Order Finite Difference Methods for Hyperbolic Problems in Deforming Domains: An Initial Investigation
}

Samira Nikkar and Jan Nordström

\begin{abstract}
A time-dependent coordinate transformation of a constant coefficient hyperbolic system of equations is considered. We use the energy method to derive well-posed boundary conditions for the continuous problem. Summation-by-Parts (SBP) operators together with a weak imposition of the boundary and initial conditions using Simultaneously Approximation Terms (SATs) guarantee energy-stability of the fully discrete scheme. We construct a time-dependent SAT formulation that automatically imposes the boundary conditions, and show that the numerical Geometric Conservation Law (GCL) holds. Numerical calculations corroborate the stability and accuracy of the approximations. As an application we study the sound propagation in a deforming domain using the linearized Euler equations.
\end{abstract}

\section{Introduction}

High order SBP-SAT schemes, can efficiently and reliably handle large problems on structured grids for reasonably smooth geometries [7, 12]. The developements within this framework, have so far dealt mostly with steady meshes while computing flow-fields around moving and deforming objects involves time-dependent meshes [3, 13]. In this paper (and the full paper [5]) we treat the time-dependent transformations in a SBP-SAT framework. To guarantee stability of the fully discrete approximation we employ the recently developed SBP-SAT technique in time $[8,4]$.

Samira Nikkar

Linköping University, SE-581 83 Linköping, e-mail: samira.nikkar@liu.se

Jan Nordström

Linköping University, SE-581 83 Linköping, e-mail: jan.nordstrom@liu.se 


\section{The continuous problem}

The following hyperbolic symmetrized constant coefficient system,

$$
V_{t}+(\hat{A} V)_{x}+(\hat{B} V)_{y}=0,(x, y) \in \Phi(t), t \in[0, T],
$$

can, with the use of the GCL [3], be rewritten as

$$
\begin{aligned}
& (J V)_{\tau}+(A V)_{\xi}+(B V)_{\eta}=0, \quad(\xi, \eta) \in \Omega, \quad \tau \in[0, T], \\
& L V=g(\tau, \xi, \eta),(\xi, \eta) \in \delta \Omega, \tau \in[0, T], \\
& V=f(\xi, \eta), \quad(\xi, \eta) \in \Omega, \quad \tau=0,
\end{aligned}
$$

through a time-dependent transformation from the Cartesian coordinates into curvilinear coordinates as

$$
x(\tau, \xi, \eta) \rightleftharpoons \xi(t, x, y), y(\tau, \xi, \eta) \rightleftharpoons \eta(t, x, y), t=\tau .
$$

In (2), $A=J \xi_{t} I+J \xi_{x} \hat{A}+J \xi_{y} \hat{B}, B=J \eta_{t} I+J \eta_{x} \hat{A}+J \eta_{y} \hat{B}$, also $\Omega=[0,1] \times[0,1]$. Moreover, $L$ is the boundary operator, $g$ is the boundary data, $f$ is the initial data. and $J=x_{\xi} y_{\eta}-x_{\eta} y_{\xi}>0$ is the determinant of the Jacobian of the transformation.

\subsection{Well-posedness}

The energy method (multiply (2) with the transpose of the solution and integrate over the domain $\Omega$ and time-interval $[0, T]$ ) is applied to (2), and the term $V_{\tau}^{T} J V+$ $V_{\xi}^{T} A V+V_{\eta}^{T} B V=0$ is added to the integral argument. Then, integration together with the use of Green-Gauss theorem gives

$$
\|V(T, \xi, \eta)\|_{J}^{2}=\|f(\xi, \eta)\|_{J}^{2}-\int_{0}^{T} \oint_{\delta \Omega} V^{T}[(A, B) \cdot n] V d s d \tau
$$

where the norm is defined by $\|V\|_{J}^{2}=\iint_{\Omega} V^{T} J V d \xi d \eta$. In (4), $n$ is the unit normal pointing outward from the $\Omega$, and $d s$ is an infinitesimal element along the boundary, $\delta \Omega$.

In order to bound the energy of the solution, boundary conditions must be applied when the matrix $C=(A, B) \cdot n$ is negative definite. We decompose $C=X \Lambda_{C} X^{T}=$ $X \Lambda_{C}^{+} X^{T}+X \Lambda_{C}^{-} X^{T}=C^{+}+C^{-}$where $\Lambda_{C}^{+}$and $\Lambda_{C}^{-}$are diagonal matrices with positive and negative eigenvalues of $C$, respectively. We choose the characteristic boundary conditions, in order to bound the energy of the solution as

$$
\left(X^{T} V\right)_{i}=\left(X^{T} V_{\infty}\right)_{i},\left(\Lambda_{C}\right)_{i i}<0,
$$

where $V_{\infty}$ is the solution at $\delta \Omega$.

The continuous energy, using (5) is estimated as 
$\|V(T, \xi, \eta)\|_{J}^{2}=\|f(\xi, \eta)\|_{J}^{2}-\int_{0}^{T} \oint_{\delta \Omega} V_{\infty}^{T} C^{-} V_{\infty} d s d \tau-\int_{0}^{T} \oint_{\delta \Omega} V^{T} C^{+} V d s d \tau$.

The estimate (6) guarantees uniqueness of the solution and existence is given by the fact that we use the correct number of boundary conditions. Hence we can summarize the results obtained so far in the following proposition.

Proposition 1. The continuous problem (2) with the boundary condition in (5) is strongly well-posed and has the bound (6).

\section{The discrete problem}

The spatial domain, $\Omega$, is a square in $\xi, \eta$ coordinates, and discretized using $N$ and $M$ nodes in $\xi$ and $\eta$ directions respectively. In time we use $\mathrm{L}$ time levels from 0 to $\mathrm{T}$.

The first derivative $u_{\xi}$ is approximated by $D_{\xi} \mathbf{u}$, where $D_{\xi}$ is a so-called SBP operator, see [10]. A multi-dimensional finite difference approximation (including the time discretization $[8,4]$ ), on SBP-SAT form, is constructed by extending the one-dimensional SBP operators in a tensor product fashion as

$$
D_{\tau}=P_{\tau}^{-1} Q_{\tau} \otimes I_{\xi} \otimes I_{\eta} \otimes I, D_{\xi}=I_{\tau} \otimes P_{\xi}^{-1} Q_{\xi} \otimes I_{\eta} \otimes I, D_{\eta}=I_{\tau} \otimes I_{\xi} \otimes P_{\eta}^{-1} Q_{\eta} \otimes I
$$

where $\otimes$ represents the Kronecker product [2]. In (7), I denotes the identity matrix with a size consistent with its position in the Kronecker product. In [5] it is shown that the operators in (7) commute.

The SBP-SAT approximation of (2) including the penalty terms for the weak boundary conditions (we only consider the boundary along which $\eta=0$, namely the south boundary, denoted by subscript s), and a weak initial condition, is constructed as

$$
\begin{aligned}
& \frac{1}{2}\left[D_{\tau}(\mathbf{J V})+\mathbf{J} D_{\tau} \mathbf{V}+\mathbf{J}_{\tau} \mathbf{V}\right]+\frac{1}{2}\left[D_{\xi}(\mathbf{A V})+\mathbf{A} D_{\xi} \mathbf{V}+\mathbf{A}_{\xi} \mathbf{V}\right]+ \\
& \frac{1}{2}\left[D_{\eta}(\mathbf{B V})+\mathbf{B} D_{\eta} \mathbf{V}+\mathbf{B}_{\eta} \mathbf{V}\right]=\tilde{P}_{i}^{-1} \Sigma_{i}(\mathbf{V}-\mathbf{f})+\tilde{P}_{s}^{-1} \Sigma_{s} \mathbf{X}_{s}^{T}\left[\mathbf{V}-V_{\infty}\right],
\end{aligned}
$$

in which the bold face of the variables corresponds to the approximated values. $\Sigma_{i}$ and $\Sigma_{s}$ are the penalty matrices for the weak initial condition and the south boundary procedure. Furthermore $\tilde{P}_{i}^{-1}=P_{\tau}^{-1} E_{0} \otimes I_{\xi} \otimes I_{\eta} \otimes I, \tilde{P}_{s}^{-1}=I_{\tau} \otimes I_{\xi} \otimes P_{\eta}^{-1} E_{0} \otimes I$, and $\mathbf{X}_{s}=\left(I_{\tau} \otimes I_{\xi} \otimes E_{0} \otimes X\right)$. Also, the vectors $V_{\infty}$ and $\mathbf{f}$ contain the boundary data at $\eta=0$ and initial data at $\tau=0$ respectively. Note that in (8), the splitting technique described in [6] is used prior to the discretizations, in order to get similar energy estimate as the one in the continuous case. 


\subsection{Stability}

The energy method (multiplying from the left with $\mathbf{V}^{T}\left(P_{\tau} \otimes P_{\xi} \otimes P_{\eta} \otimes I\right)$ ) is applied to (8) and the equation is added to its transpose. The result is

$$
\begin{aligned}
& \mathbf{V}^{T}\left(\tilde{B}_{\tau} \mathbf{J}+\tilde{B}_{\xi} \mathbf{A}+\tilde{B}_{\eta} \mathbf{B}\right) \mathbf{V}+\mathbf{V}^{T} \tilde{P}\left(\mathbf{J}_{\tau}+\mathbf{A}_{\xi}+\mathbf{B}_{\eta}\right) \mathbf{V}= \\
& \mathbf{V}^{T}\left(E_{0} \otimes P_{\xi} \otimes P_{\eta} \otimes I\right) \Sigma_{i}(\mathbf{V}-\mathbf{f})+(\mathbf{V}-\mathbf{f})^{T} \Sigma_{i}^{T}\left(E_{0} \otimes P_{\xi} \otimes P_{\eta} \otimes I\right) \mathbf{V}+ \\
& \mathbf{V}^{T}\left(P_{\tau} \otimes P_{\xi} \otimes E_{0} \otimes I\right) \Sigma_{s} \mathbf{X}_{s}^{T}\left[\mathbf{V}-\mathbf{V}_{\infty}\right]+\left[\mathbf{V}-\mathbf{V}_{\infty}\right]^{T} \mathbf{X}_{s} \Sigma_{s}^{T}\left(P_{\tau} \otimes P_{\xi} \otimes E_{0} \otimes I\right) \mathbf{V},
\end{aligned}
$$

where $\tilde{P}=\left(P_{\tau} \otimes P_{\xi} \otimes P_{\eta} \otimes I\right), \tilde{B}_{\tau}=\left[\left(Q+Q^{T}\right)_{\tau} \otimes P_{\xi} \otimes P_{\eta} \otimes I\right], \tilde{B}_{\xi}=\left[P_{\tau} \otimes(Q+\right.$ $\left.\left.Q^{T}\right)_{\xi} \otimes P_{\eta} \otimes I\right]$, and $\tilde{B}_{\eta}=\left[P_{\tau} \otimes P_{\xi} \otimes\left(Q+Q^{T}\right)_{\eta} \otimes I\right]$. The following Lemma is proved in [11].

Lemma 1. The numerical GCL holds: $\mathbf{J}_{\tau}+\mathbf{A}_{\xi}+\mathbf{B}_{\eta}=0$.

In (9), by using Lemma 1 we get

$\mathbf{V}^{T} \mathbf{J}\left(E_{L} \otimes P_{\xi \eta} \otimes \otimes I\right) \mathbf{V}=\mathbf{V}^{T}\left(E_{0} \otimes P_{\xi \eta} \otimes I\right)\left(\mathbf{J}+2 \Sigma_{i}\right) \mathbf{V}-\mathbf{f}^{T}\left(E_{0} \otimes P_{\xi \eta} \otimes I\right) \Sigma_{i} \mathbf{V}-$

$\mathbf{V}^{T}\left(E_{0} \otimes P_{\xi \eta} \otimes I\right) \Sigma_{i} \mathbf{f}+\mathbf{V}^{T}\left(P_{\tau, \xi} \otimes E_{0} \otimes I\right)\left(\mathbf{B}_{\mathbf{s}}+\Sigma_{s} \mathbf{X}_{s}^{T}+\mathbf{X}_{s} \Sigma_{s}^{T}\right) V-$

$\mathbf{V}^{T}\left(P_{\tau \xi} \otimes E_{0} \otimes I\right) \Sigma_{s} \mathbf{X}_{s}^{T}\left(\mathbf{V}_{\infty}\right)_{s}-\left(\mathbf{V}_{\infty}\right)_{s}^{T} \mathbf{X}_{s} \Sigma_{b s}^{T}\left(P_{\tau \xi} \otimes E_{0} \otimes I\right) \mathbf{V}$,

where $P_{\xi \eta}=P_{\xi} \otimes P_{\eta}, P_{\tau \xi}=P_{\tau} \otimes P_{\xi}, \mathbf{B}_{s}=\left(I_{\tau} \otimes I_{\xi} \otimes E_{0} I_{\eta} \otimes I\right) \mathbf{B}$, and $E_{0}, E_{L}$ are zero matrices except at the one entry corresponding to the initial and final time, respectively.

Proposition 2. The problem (8) is stable if $\mathbf{J}+2 \Sigma_{i} \leq 0, \Sigma_{s} \mathbf{X}_{s}^{T}+\mathbf{X}_{s} \Sigma_{s}^{T}+\mathbf{B}_{s} \leq 0$.

Proof. With zero boundary and initial data the solution at the final time is clearly bounded.

\section{Numerical experiments}

We consider the two-dimensional linearized symmetrized Euler equations in a deforming domain described by (1), where $V=[\bar{c} \rho /(\sqrt{\gamma} \bar{\rho}), u, v, T /(\bar{c} \sqrt{\gamma(\gamma-1)})]^{T}$, and $\rho, u, v, T$ and $\gamma$ are respectively the density, the velocity components in $\mathrm{x}$ and $\mathrm{y}$ directions, the temperature and the ratio of specific heats [1,14]. An equation of state in form of $\gamma p=\bar{\rho} T+\rho \bar{T}$ closes the system (1), in which the bar denotes the state around which we have linearized. Moreover the matrices in (1) are

$$
\hat{A}=\left(\begin{array}{cccc}
\bar{u} & \bar{c} / \sqrt{\gamma} & 0 & 0 \\
\bar{c} / \sqrt{\gamma} & \bar{u} & 0 & \sqrt{\frac{\gamma-1}{\gamma}} \bar{c} \\
0 & 0 & \bar{u} & 0 \\
0 & \sqrt{\frac{\gamma-1}{\gamma}} \bar{c} & 0 & \bar{u}
\end{array}\right), \hat{B}=\left(\begin{array}{cccc}
\bar{v} & 0 & \bar{c} / \sqrt{\gamma} & 0 \\
0 & \bar{v} & 0 & 0 \\
\bar{c} / \sqrt{\gamma} & 0 & \bar{v} & \sqrt{\frac{\gamma-1}{\gamma}} \bar{c} \\
0 & 0 & \sqrt{\frac{\gamma-1}{\gamma}} \bar{c} & \bar{v}
\end{array}\right) .
$$

The deforming domain is chosen to be a portion of a ring-shaped geometry where the boundaries are moving while always coinciding with a coordinate line in the 
corresponding polar coordinate system. We transform the deforming domain from Cartesian coordinates, $x, y$, into polar coordinates, $r, \phi$, and scale the polar coordinates such that $\Omega=[0,1] \times[0,1]$, see Figure 1 , as

$$
\xi(x, y, t)=\frac{r(x, y, t)-r_{0}(t)}{r_{1}(t)-r_{0}(t)}, \eta(x, y, t)=\frac{\phi(x, y, t)-\phi_{0}(t)}{\phi_{1}(t)-\phi_{0}(t)} .
$$
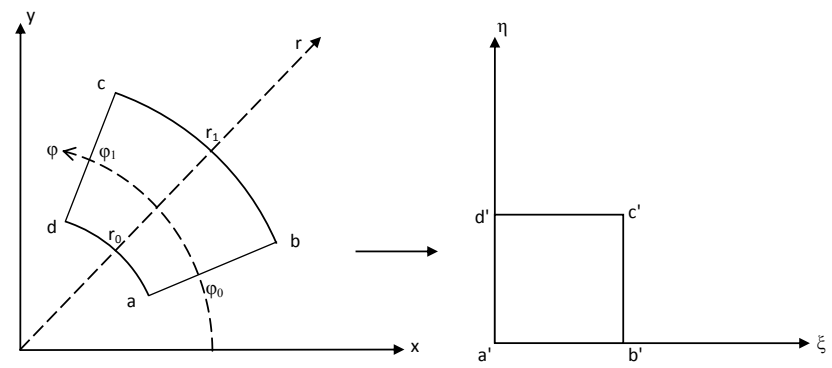

Fig. 1 A schematic of the Cartesian-polar transformation, and illustrations of $r_{0}, r_{1}, \phi_{0}$ and $\phi_{1}$; Also boundary definitions as west: $a d \rightarrow a^{\prime} d^{\prime}$, east: $b c \rightarrow b^{\prime} c^{\prime}$, south: $a b \rightarrow a^{\prime} b^{\prime}$, north: $d c \rightarrow d^{\prime} c^{\prime}$

\subsection{Order of accuracy}

We move the boundaries by the transformation

$$
\begin{aligned}
& r_{0}(t)=1-\frac{0.1}{2 \pi} \sin (2 \pi t), \phi_{0}(t)=-\frac{0.5}{2 \pi} \sin (2 \pi t) \\
& r_{1}(t)=2+\frac{0.2}{2 \pi} \sin (2 \pi t), \phi_{1}(t)=\frac{\pi}{2}+\frac{0.5}{2 \pi} \sin (2 \pi t),
\end{aligned}
$$

and construct the matrices $\hat{A}$ and $\hat{B}$ for a state where $\bar{u}=1, \bar{v}=1, \bar{\rho}=1, \bar{\gamma}=1.4$ and $\bar{c}=2$. To verify the order of accuracy of our method, we use the method of manufactured solution [9], and impose the characteristic boundary conditions as derived in (5).

The numerical solution for a scheme with SBP63 in space and SBP84 in time, converges to the exact solution at $\mathrm{T}=1$ with the convergence rate presented in table 1 . Moreover, the scheme is tested with $S B P 21$ and $S B P 42$ and the convergence rates are quantified as 2 and 3 respectively [5].

\subsection{The sound propagation application}

We consider a deforming domain where the west boundary is moving, see Figures 2 and 3. Note that these schematics are for illustration purposes only, the numerical experiments are carried out on finer meshes. The movements are defined by 


\begin{tabular}{ccccccc}
$N, M$ & 21 & 31 & 41 & 51 & 61 & 71 \\
\hline$\rho$ & 5.780 & 4.681 & 4.502 & 4.379 & 4.320 & 4.296 \\
\hline$u$ & 6.120 & 4.531 & 4.585 & 4.588 & 4.575 & 4.558 \\
\hline$v$ & 6.138 & 4.300 & 4.179 & 4.215 & 4.249 & 4.268 \\
\hline$p$ & 5.701 & 4.124 & 4.267 & 4.340 & 4.380 & 4.402
\end{tabular}

Table 1 Convergence rates at $\mathrm{T}=1$, for a sequence of mesh refinements, SBP63 in space, SBP84 in time $(\mathrm{L}=201)$

$$
\begin{aligned}
& r_{0}(t)=1+\sin (4 \pi t) /(4 \pi), \phi_{0}(t)=\pi / 4 \\
& r_{1}(t)=5, \phi_{1}(t)=3 \pi / 4
\end{aligned}
$$

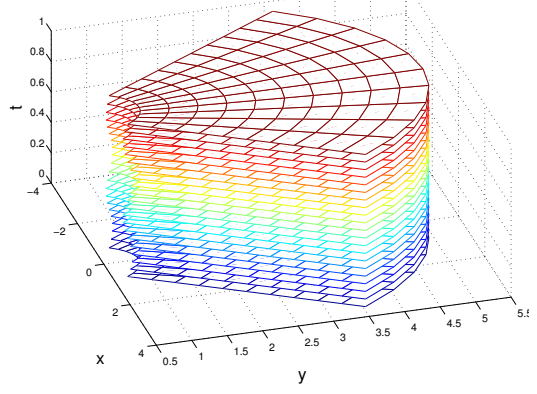

Fig. 2 A schematic of the deforming mesh at different times, sound propagation.

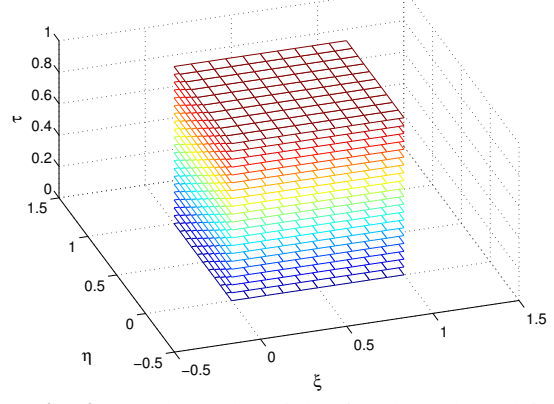

Fig. 3 A schematic of the fixed mesh at different times, sound propagation.

We choose $\gamma=1.4, \bar{c}=2, \bar{\rho}=1$ and manufacture $\bar{u}$ and $\bar{v}$ such that the mean flow satisfies the solid wall no-penetration condition at the moving boundary by

$$
\bar{u}=x_{\tau} / \exp (\xi), \bar{v}=y_{\tau} / \exp (\xi) .
$$

Consider the eigenvalue matrix, $C=X \Lambda X^{T}$ at the west boundary, in which $\Lambda=$ $\mathscr{R}_{1} \operatorname{diag}(\hat{\omega}, \hat{\omega}, \hat{\omega}-\bar{c}, \hat{\omega}+\bar{c})$, where $\hat{\omega}=-\left(J \xi_{t}+J \xi_{x} \bar{u}_{b}+J \xi_{y} \bar{v}_{b}\right) / \mathscr{R}_{1}$ and $\mathscr{R}_{1}=$ $\sqrt{\left(J \xi_{x}\right)^{2}+\left(J \xi_{y}\right)^{2}}$. The no-penetration condition for the mean flow at the moving boundary results in $\hat{\omega}=0$, which takes (6) to

$$
\|V(T, \xi, \eta)\|_{J}^{2}=\|f(\xi, \eta)\|^{2}-\int_{0}^{T} \int_{0}^{1} \bar{c}\left(\tilde{v}_{4}^{2}-\tilde{v}_{3}^{2}\right) d \eta+\mathrm{BT} .
$$

In (16), $\tilde{V}=X^{T} V=\left[\tilde{v}_{1}, \tilde{v}_{2}, \tilde{v}_{3}, \tilde{v}_{4}\right]^{T}$, and $B T$ is the contribution at the other boundaries. Any boundary condition of the form $\tilde{v}_{3}= \pm \tilde{v}_{4}$ is well-posed. We choose $\tilde{v}_{3}+\tilde{v}_{4}=0$, which is the no-penetration boundary condition. Also we impose characteristic boundary conditions with zero data at the other boundaries, and initialize the solution with zero data for density and velocities, together with an initial pressure pulse centered at $(-1.5,3.5)$. We have used $N=M=50, L=100$ and SBP42 in space and time. The velocity field at two different time levels, with non-penetrating flow close to the solid wall, are presented in Figures 4-7. 


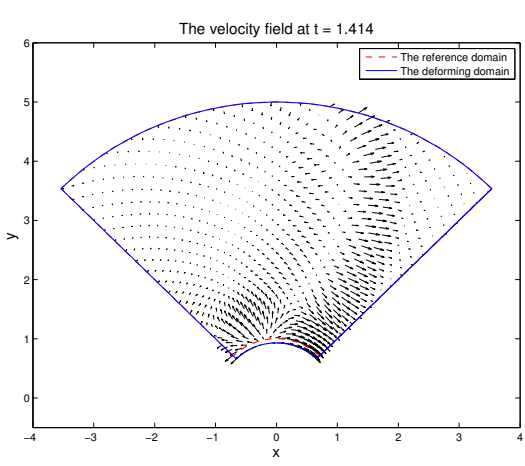

Fig. 4 The global velocity field.

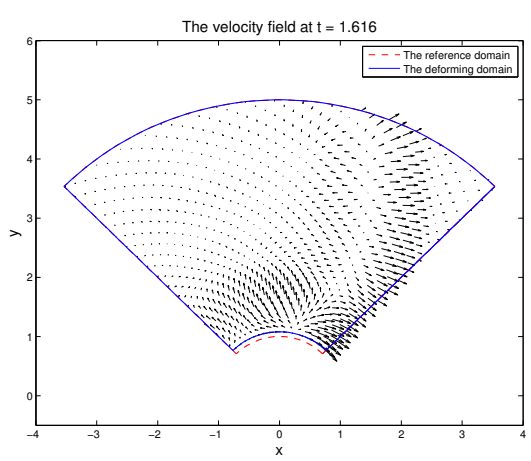

Fig. 6 The global velocity field.

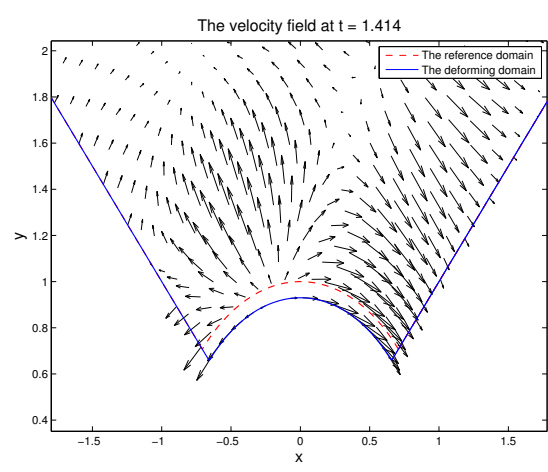

Fig. 5 A blow-up of the velocity field.

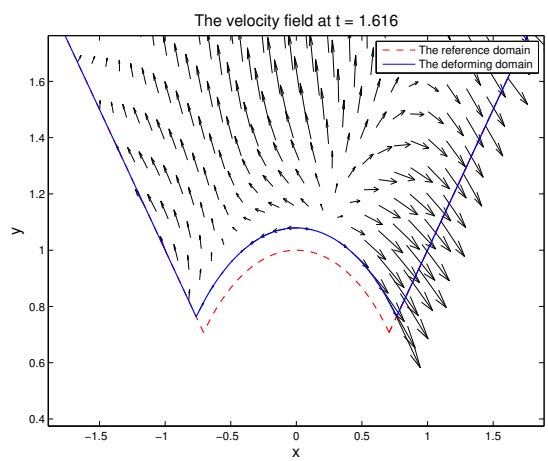

Fig. 7 A blow-up of the velocity field.

The reference domain in Figures 4-7 illustrate the movements of the south boundary relative to its initial location. As seen in the figures, the flow stays tangential to the moving solid boundary all the time, as it should for an Euler solution.

\section{Summary and conclusions}

We have considered a constant coefficient hyperbolic system of equations in timedependent curvilinear coordinates. The system is transformed into a fixed coordinate frame, resulting in variable coefficient system. We show that the energy method applied to the transformed systems together with time-dependent appropriate boundary conditions leads to strongly well-posed problem.

By using a special splitting technique, summation-by-parts operators in space and time, weak imposition of the boundary and initial conditions and the discrete energy method, a fully-discrete strongly stable and high order accurate numerical 
scheme is constructed. The fully-discrete energy estimate is similar to the continuous one with small added damping terms. Furthermore, by the use of SBP operators in time, the Geometric Conservation Law is shown to hold numerically.

We have tested the scheme for high order accurate SBP operators in space and time using the method of manufactured solution. Numerical calculations corroborate the stability and accuracy of the fully-discrete approximations. Finally, as an application, sound propagation by the linearized Euler equations in a deforming domain is illustrated.

\section{References}

1. Abrabanel, S., Gottleib, D.: Optimal time splitting for two- and three-dimensional NavierStokes equations with mixed derivatives, Journal of Computational Physics, 41, 1-43, (1981).

2. Charles F. Van Loan: The ubiquitous Kronecker product, Journal of Computational and Applied Mathematics, 123, 85-100, (2000).

3. Farhat, C., Geuzaine, P., Grandmont, C.: The discrete geometric conservation law and the nonlinear stability of ALE schemes for the solution of flow problems on moving grids, Journal of Computational Physics, 174, (2001).

4. Lundquist. T., Nordström, J.: The SBP-SAT Technique for Initial Value Problems, Journal of Computational Physics, 270, 86-104, (2014).

5. Nikkar, S., Nordström, J., Fully Discrete Energy Stable High Order Finite Difference Methods for Hyperbolic Problems in Deforming Domains, LiTH- MAT-R, 2014:15, 2014, Department of Mathematics, Linköping University.

6. Nordström, J.: Conservative Finite Difference Formulations, Variable Coefficients, Energy Estimates and Artificial Dissipation, Journal of Scientific Computing, 29, (2006).

7. Nordström, J., Carpenter, H.: High-order finite difference methods, multidimensional linear problems and curvilinear coordinates, Journal of Computational Physics, 173, (2001).

8. Nordström, J., Lundquist. T.: Summation-By-Parts in Time, Journal of Computational Physics, 251, 487-499, (2013).

9. Salari, K.: Code Verification by the Method of Manufactured Solutions, doi: 10.2172/759450.

10. Strand B.: Summation by Parts for Finite Difference Approximations of d/dx, Journal of Computational Physics, 110, (1994).

11. Abe, Y., Izuka, N., Nonomura, T., Fuji, K.: Symmetric-conservative metric evaluations for higher-order finite difference scheme with the GCL identities on the three-dimensional moving and deforming mesh, ICCFD7 (2012).

12. Svärd, M., Nordström, J.: A stable high-order finite difference scheme for the compressible Navier Stokes equations: no-slip wall boundary conditions, Journal of Computational Physics, 227 (10), (2008).

13. Thomas, P.D., Lombard, C.K.: Geometric Conservation Law and Its Application to Flow Computations on Moving Grids, AIAA Journal, 17, (1979).

14. Turkel, E.: Symmetrization of the fluid dynamics matrices with applications, Math. Comp., 27, 729-736, (1973). 